\title{
HYBRID MAXIMUM A POSTERIORI ESTIMATION UNDER COMMUNICATION CONSTRAINTS
}

\author{
Esha D. Nerurkar and Stergios I. Roumeliotis
}

\author{
Department of Computer Science and Engineering, University of Minnesota, USA
}

\begin{abstract}
We consider the problem of joint-state estimation for mobile wireless sensor networks (WSN) using noisy analog observations from spatially-distributed sensors. Due to communication bandwidth constraints, sensors can transmit only quantized observations. As opposed to existing estimators that process either only quantized or only analog observations, we develop a Maximum A Posteriori-based hybrid estimation framework that enables each sensor to utilize its own local analog observations as well as quantized observations received from other sensors to improve estimation accuracy.
\end{abstract}

Index Terms - Distributed MAP estimation, wireless sensor network, communication constraints

\section{INTRODUCTION AND RELATED WORK}

For sensor localization in GPS-denied environments, mobile WSN (e.g., mobile robots) combine noisy observations of motion (e.g., velocity) and relative position (e.g., distance/bearing to each other), from all sensors, to accurately estimate their joint-state, i.e., their positions and velocities. Since the sensors are spatially distributed, transmitting these analog observations incurs substantial communication overhead. In this work, we focus on WSN localization in the presence of severe communication-bandwidth constraints, where each sensor can transmit only a few bits per analog observation. Thus, every sensor carries out lossy quantization of its observations and the commonly-used estimation frameworks for analog observations [e.g., the Kalman Filter (KF) or the Maximum A Posteriori (MAP) estimator] have to be modified to accommodate these quantized observations.

While there exists a large body of work on parameter estimation (either deterministic [1, 2, 3] or random variable [4, $5,6]$ ) for WSN, we consider approaches that were developed to estimate random processes, as is the case in sensor localization. The Sign-of-Innovation Kalman filter, for estimating stochastic, dynamic processes, has been proposed [7], where the measurement innovation ${ }^{1}$, instead of the actual analog

\footnotetext{
This work was supported by the University of Minnesota through the Digital Technology Center (DTC), and AFOSR (FA9550-10-1-0567).

${ }^{1}$ Measurement innovation is the difference between the actual and the estimated (by the estimator) measurement.
}

measurement ${ }^{2}$, is quantized to a single bit. When $f \geq 1$ bits are available, this approach has been extended in [8] to the batch and iteratively quantized KF, where with $f=4$ bits, the performance is almost indistinguishable from that of the standard KF. These estimators are derived for linear, Gaussian motion and measurement models and they approximate the posterior probability density function by a Gaussian ${ }^{3}$ after each measurement update, to reduce computational complexity. For nonlinear models, in order to mitigate the effect of linearization and Gaussian approximations carried out above, extensions of this approach to the MAP estimator (that acts as a smoother), for single bit (QMAP) and multiple bits (batch and iteratively quantized BQMAP and IQMAP, respectively), are presented in $[9,10,11]$.

The quantized innovation estimators discussed above use the multi-centralized (MC) architecture (robust to single point failures) where each sensor broadcasts all its observations and every sensor locally processes observations from the entire network to generate joint-state estimates. However, the quantization rules used by these estimators depend upon the computed state estimates, via the measurement innovation, and thus all sensors have to maintain identical estimators to ensure estimation consistency. Hence, each sensor is forced to use quantized versions of its own locally-available, analog observations for estimation, thus discarding valuable information that can be used to improve localization accuracy.

To overcome this drawback, we have introduced a hybrid estimation framework in [12, 13], for Minimum Mean Squared Error (MMSE) estimation (filtering), that enables each sensor to incorporate its locally-available, analog observations in the estimation process. Specifically, each sensor maintains two local estimators (see Fig. 1): (i) a quantized (Q) estimator that processes quantized observations from all sensors, including itself, and (ii) a hybrid $(\mathrm{H})$ estimator that processes its own analog observations along with the quantized observations from other sensors. In this paper, we extend the hybrid estimation framework to MAP estimation and derive a $(\mathrm{H})$ estimator, called H-BQMAP, that by using its own analog observations can outperform the existing BQMAP, that uses only quantized observations.

\footnotetext{
${ }^{2}$ We use measurement and observation interchangeably.

${ }^{3}$ Note that due to the nonlinearity of the quantization operation, the posterior pdf is not, in general, a Gaussian.
} 


\section{PROBLEM FORMULATION}

Consider a mobile WSN consisting of $N$ sensor nodes. The motion (process) model for the WSN is given by the following linear, discrete-time, dynamic system driven by system noise:

$$
\mathbf{x}_{k}=\mathbf{F}_{k-1} \mathbf{x}_{k-1}+\mathbf{G}_{k-1} \mathbf{w}_{k-1}, p\left(\mathbf{x}_{0}\right) \sim \mathcal{N}\left(\mathbf{x}(0), \mathbf{P}_{0}\right)
$$

where, $\mathbf{w}_{k}$ is the zero-mean, white, Gaussian, and uncorrelated system noise at time-step $k$ with covariance given by $E\left[\mathbf{w}_{k} \mathbf{w}_{l}^{T}\right]=\delta_{k l} \mathbf{Q}_{k}$. Here, $\mathbf{x}_{k}=\left[\mathbf{x}_{k}^{1^{T}}, \mathbf{x}_{k}^{2^{T}}, \ldots, \mathbf{x}_{k}^{N^{T}}\right]^{T}$, is the joint-state of the sensor network and $\mathbf{x}_{k}^{i}$ denotes the state ${ }^{4}$ of each individual sensor $i$ at time-step $k, k=$ $0, \ldots, K . p\left(\mathbf{x}_{0}\right)$ is the prior on the sensors' initial states.

Sensor $i$ obtains $M_{k}^{i}$ scalar, analog measurements at timestep $k$. Sensor $i$ 's measurement model, $i=1, \ldots, N$, is:

$$
z_{k m}^{i}=\mathbf{h}_{k m}^{i^{T}} \mathbf{x}_{k}+v_{k m}^{i}, m=1, \ldots, M_{k}^{i}
$$

where $v_{k m}^{i}$ is zero-mean, white, Gaussian, and uncorrelated measurement noise with $E\left[v_{k m}^{i} v_{l n}^{i}\right]=\delta_{k l, m n} \sigma_{k m}^{i^{2}}$ and $E\left[v_{k m}^{i} v_{l n}^{j}\right]=0, \forall j \neq i, j=1, \ldots, N$. The noise terms in the process and measurement models are independent. We assume that: (i) the process and measurement models are shared a priori by all sensors before deployment, and (ii) each sensor can communicate with the team at every time step. The linear models (1)-(2) are used to simplify the mathematical derivations. In real-world scenarios, the linearized system, obtained from the underlying non-linear models, will be used. Moreover, a single vector-valued measurement can be decomposed into multiple scalar measurements using pre-whitening [14] and then processed using the above formulation. Lastly, in order to simplify the notation in this paper, from now onwards, we assume that each sensor $i$ obtains only a single, scalar, analog measurement, $z_{k}^{i}$, at time-step $k$. The generalization to $M_{k}^{i}$ measurements is straightforward.

\subsection{Real vs. Quantized Measurements}

In the absence of communication-bandwidth constraints, the Maximum A Posteriori (MAP) estimate, $\hat{\mathbf{x}}_{0: K}$, of all sensors' states from time-step 0 to $K$, given all analog measurements up to time-step $K, \mathbf{z}_{0: K}$, is calculated as:

$$
\begin{aligned}
& \hat{\mathbf{x}}_{0: K}=\arg \max p\left(\mathbf{x}_{0: K} \mid \mathbf{z}_{0: K}\right) \\
& =\arg \max \frac{1}{p\left(\mathbf{z}_{0: K}\right)} p\left(\mathbf{z}_{0: K} \mid \mathbf{x}_{0: K}\right) p\left(\mathbf{x}_{0: K}\right) \\
& =\arg \max \frac{1}{p\left(\mathbf{z}_{0: K}\right)} \prod_{k=0}^{K} \prod_{i=1}^{N} p\left(z_{k}^{i} \mid \mathbf{x}_{k}\right) \prod_{k=0}^{K-1} p\left(\mathbf{x}_{k+1} \mid \mathbf{x}_{k}\right) \cdot p\left(\mathbf{x}_{0}\right)
\end{aligned}
$$

Here, $\mathbf{z}_{0: K}=\left[\left(\mathbf{z}_{0: K}^{1}\right)^{T}, \ldots,\left(\mathbf{z}_{0: K}^{N}\right)^{T}\right]^{T}$ where, $\mathbf{z}_{0: K}^{i}=$ $\left[z_{0}^{i}, \ldots, z_{K}^{i}\right]^{T}, i=1, \ldots, N$. Under the Gaussian noise assumption for the linear system (1)-(2), the conditional pdfs

\footnotetext{
${ }^{4}$ For example, for the sensor localization task, the joint-state consists of the positions and velocities of all the sensors.
}

in (3) and hence the posterior pdf $p\left(\mathbf{x}_{0: K} \mid \mathbf{z}_{0: K}\right)$ are Gaussian. Therefore, the optimization problem in (3) can be formulated as a Weighted Least Squares and solved using standard methods [15] such as normal equations, QR decomposition, etc. However, note that the real-valued measurements, $\mathbf{z}_{0: K}$, from all sensors, are required for solving the optimization problem in (3) and each sensor $i$ has to broadcast its measurements, $\mathbf{z}_{0: K}^{i}$, to the WSN.

On the contrary, in WSNs with severe power and communication bandwidth limitations, each sensor can communicate only $f \geq 1$ bits per analog measurement. Therefore, sensor $i$ must quantize its analog measurement, $z_{k}^{i} \in \mathbb{R}$, to $b_{k}^{i} \in \mathcal{B}$, $\mathcal{B}:=\left\{1, \ldots, 2^{f}\right\}$ using a quantization rule $\mathbf{q}[\cdot]$ of the form:

$$
b_{k}^{i}=\mathbf{q}\left[z_{k}^{i}\right], \text { where } \mathbf{q}: \mathbb{R} \rightarrow \mathcal{B}
$$

Thus, the MAP estimate of the sensors' states, $\hat{\mathbf{x}}_{0: K}^{Q}$, given quantized measurements, $\mathbf{b}_{0: K}$, from all sensors, up to timestep $K$ is calculated as:

$$
\begin{aligned}
& \hat{\mathbf{x}}_{0: K}^{Q}=\arg \max p\left(\mathbf{x}_{0: K} \mid \mathbf{b}_{0: K}\right) \\
& =\arg \max \frac{1}{p\left(\mathbf{b}_{0: K}\right)} \prod_{k=0}^{K} \prod_{i=1}^{N} p\left(b_{k}^{i} \mid \mathbf{x}_{k}\right) \prod_{k=0}^{K-1} p\left(\mathbf{x}_{k+1} \mid \mathbf{x}_{k}\right) \cdot p\left(\mathbf{x}_{0}\right)
\end{aligned}
$$

Importantly, note that due to the nonlinear quantization operation in (4), the conditional pdf $p\left(b_{k}^{i} \mid \mathbf{x}_{k}\right)$ and hence the resulting posterior pdf are no longer Gaussian even for the linear process and measurement models.

In the BQMAP estimator $[10,11]$, that generates these bits by quantizing the measurement innovation, $z_{k}^{i}-\mathbf{h}_{k}^{i^{T}} \hat{\mathbf{x}}_{\tau k}^{Q}$, it has been shown that the resulting pdf $p\left(\mathbf{x}_{0: K} \mid \mathbf{b}_{0: K}\right)$ in (5) is log-concave in $\mathbf{x}_{0: K}$. Hence, we can find a unique, globally optimum solution for the BQMAP estimator. Here, the state estimate $\hat{\mathbf{x}}_{\tau k}^{Q}$ is chosen to be the latest available MAP estimate (using quantized measurements only) for $\mathbf{x}_{k}$. However, a shortcoming of this approach is that even though each sensor $i$ has access to its own analog measurements, $\mathbf{z}_{0: K}^{i}$, the quantized-innovation BQMAP estimator forces it to discard this information and process only the corresponding quantized measurements, $\mathbf{b}_{0: K}^{i}$. This is because the quantized measurement, $b_{k}^{i}$, depends upon the estimated measurement, $\mathbf{h}_{k}^{i^{T}} \hat{\mathbf{x}}_{\tau k}^{Q}$, which has to be identical for all sensors to ensure consistent estimation. Therefore, all sensors have to process the same set of measurements, so that they can generate identical state estimates, $\hat{\mathbf{x}}_{\tau k}^{Q}$. In Fig. 1, when the shaded Hybrid Estimators do not exist, we obtain the BQMAP.

\section{HYBRID ESTIMATION FRAMEWORK}

In [12, 13], we introduced a hybrid estimation framework for filtering that enables each sensor to obtain MMSE state estimates (under Gaussian assumption) by processing local analog and remote quantized measurements. In this work, 


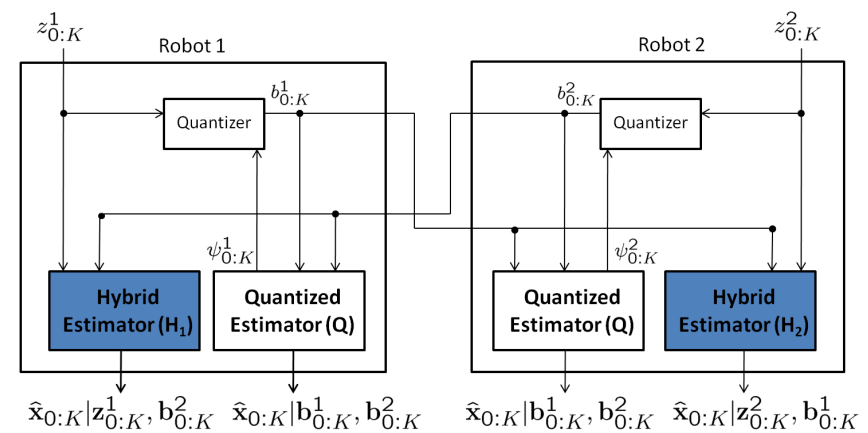

Fig. 1. Hybrid Estimation framework. Here, $\psi_{k}^{i}=\mathbf{h}_{k}^{i^{T}} \hat{\mathbf{x}}_{\tau k}^{Q}$, $k=0, \ldots, K, i=1,2$, and using the quantization rule in (6), the H- and Q-estimators correspond to the H-BQMAP and BQMAP, respectively.

we focus on MAP estimation since for the nonlinear process/measurement models in real-world systems, the MAP estimator acts as a smoother and mitigates linearization errors, hence improving estimation accuracy. To achieve this, we propose that each sensor $i$ maintains two estimators (see Fig. 1): (1) a quantized (Q) estimator that processes quantized measurements from all sensors including itself, i.e., $\hat{\mathbf{x}}_{0: K}^{Q}=$ $\arg \max p\left(\mathbf{x}_{0: K} \mid \mathbf{b}_{0: K}^{q \neq i}, \mathbf{b}_{0: K}^{i}\right)$, and (2) a hybrid (H) estimator that processes its own real-valued measurements and quantized measurements from the other sensors in the team, i.e., $\hat{\mathbf{x}}_{0: K}^{H_{i}}=\arg \max p\left(\mathbf{x}_{0: K} \mid \mathbf{b}_{0: K}^{q \neq i}, \mathbf{z}_{0: K}^{i}\right), q=1, \ldots, N$. The estimates generated by the Q-estimator are identical for all sensors since each sensor processes identical measurements, $\mathbf{b}_{0: K}=\left\{\mathbf{b}_{0: K}^{q \neq i}, \mathbf{b}_{0: K}^{i}\right\}$. Therefore, they are used in the hybrid estimation framework for generating identical quantization thresholds for all sensors, as will be shown in the next section. On the contrary, the estimates generated by the $\mathrm{H}$ estimator are different for each sensor, since each sensor processes a different set of quantized and analog measurements. In the next section, we will derive the Q-and H-estimators, BQMAP and H-BQMAP, respectively, for the quantization scenario where the bandwidth availability ( $f$ bits per analog measurement) is known a priori.

\subsection{Quantization Rule}

Since sensor $j$ is pre-informed about the availability of $f \geq 1$ bits for communicating its analog measurement $z_{k}^{j} \in \mathbb{R}$, sensor $j$ partitions the observation space $\mathbb{R}$ into $2^{f}$ intervals. The interval $\mathcal{R}_{k}^{j}(n):=\left[\tau_{k}^{j}(n), \tau_{k}^{j}(n+1)\right)$, where $\tau_{k}^{j}(n)$ are the quantization thresholds, $n \in \mathcal{B}:=\left\{1, \ldots, 2^{f}\right\}, \tau_{k}^{j}(1)=$ $-\infty, \tau_{k}^{j}\left(2^{f}+1\right)=\infty$, and $\tau_{k}^{j}(n)<\tau_{k}^{j}(n+1)$. The quantization rule, based on the measurement innovation, has the form ${ }^{5}[8]$ :

\footnotetext{
${ }^{5} \mathrm{We}$ assume a round-robin scheduling algorithm where the quantized measurements are generated and processed sequentially, based on sensor ids. Therefore, sensor $i$ generates and communicates its quantized measurement, $b_{k}^{i}$, before sensor $(i+1)$. Moreover, all sensors in the team process $b_{k}^{i}$ to
}

$$
b_{k}^{j}=n, \text { iff } \tau_{k}^{j}(n)<z_{k}^{j}-\mathbf{h}_{k}^{j^{T}} \hat{\mathbf{x}}_{\tau k}^{Q} \leq \tau_{k}^{j}(n+1)
$$

where, $\hat{\mathbf{x}}_{\tau k}^{Q}$ is the BQMAP's latest available estimate for the state $\mathbf{x}_{k}$. The thresholds $\tau_{k}^{j}(n)$ are defined as:

$$
\tau_{k}^{j}(n)=\Delta(n) \sqrt{\left(\mathbf{h}_{k}^{j^{T}} \mathbf{P}_{k} \mathbf{h}_{k}^{j}+\sigma_{k}^{j^{2}}\right)}
$$

where $\mathbf{P}_{k}$ is the covariance of the estimate $\hat{\mathbf{x}}_{\tau k}^{Q}$ and $\Delta(n)$ corresponds to the Lloyd-Max quantization thresholds [16, 17]. This is equivalent to quantizing the measurement innovation, $z_{k}^{j}-\mathbf{h}_{k}^{j^{T}} \hat{\mathbf{x}}_{\tau k}^{Q}$, with minimum MSE distortion [8]. Note that sensor $j$ uses its BQMAP's estimate in (6) that is identical for all sensors. This enables all sensors to correctly process/decode the quantized measurement, since this estimate is needed in the design of the H-BQMAP and BQMAP (see (8), (9), and [11]). Sensor $j$ cannot use its H-BQMAP's estimate since it depends upon its local analog measurements, $\mathbf{z}_{0 \text { :K }}^{j}$, which are unavailable to the rest of the sensors.

\subsection{BQMAP and H-BQMAP Estimators}

For the batch quantization rule from (6), we now derive the resulting $(\mathrm{Q})$ and $(\mathrm{H})$ MAP estimators for the hybrid estimation framework. Note that the Q-estimator, by definition, is identical to the BQMAP in [10] and is not described here to conserve space. Before proceeding, we first calculate the conditional probability $p\left(b_{k}^{q}=n \mid \mathbf{x}_{k}\right)$ as follows:

$$
\begin{gathered}
p\left(b_{k}^{q}=n \mid \mathbf{x}_{k}\right)=\operatorname{Pr}\left\{\tau_{k}^{q}(n)<z_{k}^{q}-\mathbf{h}_{k}^{q^{T}} \hat{\mathbf{x}}_{\tau k}^{Q} \leq \tau_{k}^{q}(n+1) \mid \mathbf{x}_{k}\right\} \\
=\left(\mathrm{Q}\left[\frac{\tau_{k}^{q}(n)-\mathbf{h}_{k}^{q^{T}}\left(\mathbf{x}_{k}-\hat{\mathbf{x}}_{\tau k}^{Q}\right)}{\sigma_{k}^{q}}\right]\right. \\
\left.-\mathrm{Q}\left[\frac{\tau_{k}^{q}(n+1)-\mathbf{h}_{k}^{q^{T}}\left(\mathbf{x}_{k}-\hat{\mathbf{x}}_{\tau k}^{Q}\right)}{\sigma_{k}^{q}}\right]\right)
\end{gathered}
$$

where, $\mathrm{Q}[x]=\int_{x}^{\infty} \frac{1}{\sqrt{2 \pi}} \exp \left(-u^{2} / 2\right) d u$, is the Gaussian tail probability and we use the fact that the conditional pdf $p\left(z_{k}^{q} \mid \mathbf{x}_{k}\right) \sim \mathcal{N}\left(\mathbf{h}_{k}^{q T} \mathbf{x}_{k}, \sigma_{k}^{q^{2}}\right)$. We now present the derivation for sensor $i$ 's H-estimator, the H-BQMAP. The MAP estimate, $\hat{\mathbf{x}}_{0: K}^{H_{i}}$, computed by sensor $i$ 's H-estimator using (i) its own analog measurements, $\mathbf{z}_{0: K}^{i}$, and (ii) quantized bits, $\mathbf{b}_{0: K}^{q \neq i}, q=1, \ldots, N$, received from other sensors in the team is given by:

$$
\begin{aligned}
\hat{\mathbf{x}}_{0: K}^{H_{i}}=\arg \max p\left(\mathbf{x}_{0: K} \mid \mathbf{b}_{0: K}^{q \neq i}, \mathbf{z}_{0: K}^{i}\right) \\
=\arg \max \left(\frac{1}{p\left(\mathbf{b}_{0: K}^{q \neq i}, \mathbf{z}_{0: K}^{i}\right)} \prod_{k=0}^{K} p\left(z_{k}^{i} \mid \mathbf{x}_{k}\right) \prod_{k=0}^{K} \prod_{q=1, q \neq i}^{N} p\left(b_{k}^{q} \mid \mathbf{x}_{k}\right)\right. \\
\left.\quad \times \prod_{k=0}^{K-1} p\left(\mathbf{x}_{k+1} \mid \mathbf{x}_{k}\right) \cdot p\left(\mathbf{x}_{0}\right)\right)
\end{aligned}
$$

obtain $\hat{\mathbf{x}}_{0: k, i}^{Q}$, before sensor $(i+1)$ generates its quantized measurement. 
where we obtain (9) using Bayes' rule. For the linear model of (1)-(2), $p\left(\mathbf{x}_{k+1} \mid \mathbf{x}_{k}\right) \sim \mathcal{N}\left(\mathbf{F}_{k} \mathbf{x}_{k}, \mathbf{G}_{k} \mathbf{Q}_{k} \mathbf{G}_{k}^{T}\right)$ and $p\left(z_{k}^{i} \mid \mathbf{x}_{k}\right) \sim \mathcal{N}\left(\mathbf{h}_{k}^{i T} \mathbf{x}_{k}, \sigma_{k}^{i^{2}}\right)$. Therefore, the above equation can be written as:

$$
\begin{aligned}
& \hat{\mathbf{x}}_{0: K}^{H_{i}}=\arg \max p\left(\mathbf{x}_{0: K} \mid \mathbf{b}_{0: K}^{q \neq i}, \mathbf{z}_{0: K}^{i}\right) \\
& =\arg \max \left(\prod_{k=0}^{K} \mathcal{N}\left(\mathbf{h}_{k}^{i T} \mathbf{x}_{k}, \sigma_{k}^{i^{2}}\right) \prod_{k=0}^{K} \prod_{q=1, q \neq i}^{N} p\left(b_{k}^{q} \mid \mathbf{x}_{k}\right)\right. \\
& \left.\quad \times \prod_{k=0}^{K-1} \mathcal{N}\left(\mathbf{F}_{k} \mathbf{x}_{k}, \mathbf{G}_{k} \mathbf{Q}_{k} \mathbf{G}_{k}^{T}\right) \mathcal{N}\left(\mathbf{x}(0), \mathbf{P}_{0}\right)\right) \\
& =\arg \min \left(\sum_{k=0}^{K} \frac{1}{2}\left\|z_{k}^{i}-\mathbf{h}_{k}^{i T} \mathbf{x}_{k}\right\|_{\sigma_{k}^{i-2}}^{2}-\sum_{k=0}^{K} \sum_{q=1, q \neq i}^{N} \log p\left(b_{k}^{q} \mid \mathbf{x}_{k}\right)\right. \\
& \left.+\sum_{k=0}^{K-1} \frac{1}{2}\left\|\mathbf{x}_{k+1}-\mathbf{F}_{k} \mathbf{x}_{k}\right\|_{\left(\mathbf{G}_{k} \mathbf{Q}_{k} \mathbf{G}_{k}^{T}\right)^{-1}}^{2}+\frac{1}{2}\left\|\mathbf{x}_{0}-\mathbf{x}(0)\right\|_{\mathbf{P}_{0}^{-1}}^{2}\right)
\end{aligned}
$$

where $p\left(b_{k}^{q} \mid \mathbf{x}_{k}\right)$ is as defined in (8). The $p\left(\mathbf{b}_{0: K}^{q \neq i}, \mathbf{z}_{0: K}^{i}\right)$ is a normalizing constant that is neglected during optimization. Next, we prove a very important property for the posterior pdf $p\left(\mathbf{x}_{0: K} \mid \mathbf{b}_{0: K}^{q \neq i}, \mathbf{z}_{0: K}^{i}\right)$.

Lemma 1. The posterior pdf of the H-BQMAP in (9) is logconcave.

Proof. Consider the innovation term $r_{k}^{q}=z_{k}^{q}-\mathbf{h}_{k}^{q^{T}} \hat{\mathbf{x}}_{\tau k}^{Q}$, where $p\left(r_{k}^{q} \mid \mathbf{x}_{k}\right) \sim \mathcal{N}\left(\mathbf{h}_{k}^{q^{T}}\left(\mathbf{x}_{k}-\hat{\mathbf{x}}_{\tau k}^{Q}\right), \sigma_{k}^{q^{2}}\right)$. This conditional pdf is log-concave in both arguments. Therefore, $p\left(b_{k}^{q} \mid \mathbf{x}_{k}\right)=$ $\int_{\tau_{k}^{q}(n)}^{\tau_{k}^{q}(n+1)} p\left(r_{k}^{q} \mid \mathbf{x}_{k}\right) d r_{k}^{q}$ is also log-concave since it involves the integral over a convex set of a log-concave pdf [18]. Thus, both the Gaussian pdfs and (8) are log-concave. Also, logconcavity is closed under multiplication and preserved under linear transformation of the argument $[19,10]$.

We conclude that, due to the log-concavity of this posterior pdf, the optimization problem in (10) is nonlinear but convex. Hence, it is guaranteed to converge to the global optimum and can be solved using efficient convex optimization techniques [19] such as Newton's method, the interior point methods, etc. Moreover, its solution, i.e., the MAP estimate, is unique and the global optimum.

\section{SIMULATION RESULTS}

The simulation set up consists of two sensors deployed in 1D. The motion model for these sensors is given by a constant velocity statistical model [20]. Each sensor obtains noisy measurements for its own velocity, $v_{m}$, and distance, $d_{m}$, to the other sensor, with the noise modeled as zero-mean, white Gaussian with std. dev. $\sigma_{v_{m}}=0.01 \mathrm{~m} / \mathrm{s}$ and $\sigma_{d_{m}}=0.037 \mathrm{~m}$, respectively. We compare the performance of the proposed $\mathrm{H}$ estimator, H-BQMAP, using $f=\{1,4\}$ bits per analog measurement, with: (1) the Q-estimator (local and remote quantized measurements), BQMAP, and (2) the real-valued MAP that uses analog measurements from all sensors and hence is our benchmark. Fig. 2 shows the root mean squared error (RMSE) in the position and velocity estimates for these estimators, averaged over the 2 sensors and 10 Monte Carlo trials. Since the estimates generated by the H-BQMAP are different for each sensor, the RMSE for H-BQMAP is also averaged over estimators maintained by each sensor. As evident from Fig. 2, the estimates generated by the H-BQMAP are more accurate that the BQMAP, irrespective of the number of quantization bits considered. This is expected since the H-BQMAP includes local analog measurements in the estimation process. Specifically, when $f=1$, the H-BQMAP is significantly more accurate than the BQMAP. Overall, the error in the estimates ${ }^{6}$ decreases as we increase the number of quantization bits (results for $f=\{2,3\}$ bits not shown for clarity) and by communicating as few as 4 bits per analog measurement, both the H-BQMAP and BQMAP are able to achieve accuracy very close to that of the real MAP estimator.

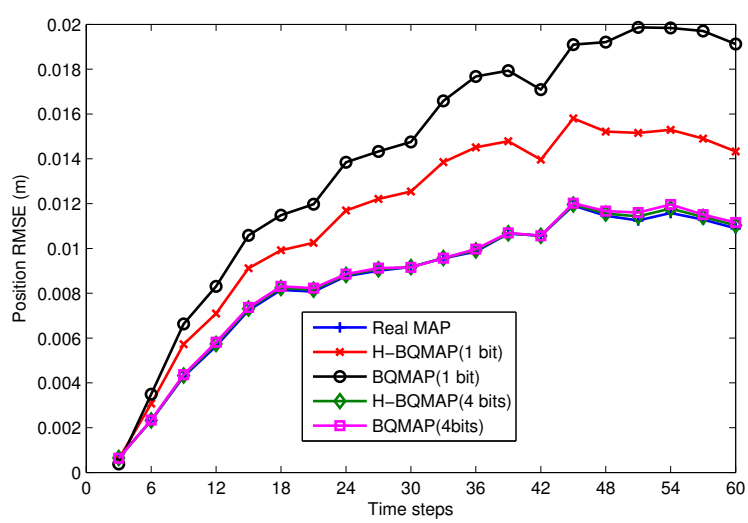

(a) Comparison of position RMSE.

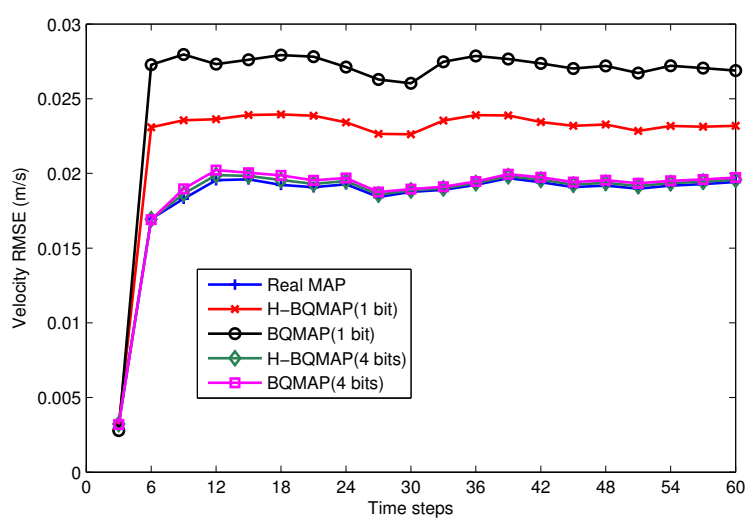

(b) Comparison of velocity RMSE.

Fig. 2. Comparison of position and velocity RMSE for the real-valued, quantized and hybrid MAP estimators.

\footnotetext{
${ }^{6}$ Note that this gain in estimation accuracy comes at the cost of increased processing, as each sensor has to maintain both H- and Q-MAP estimators (see Fig. 1).
} 


\section{REFERENCES}

[1] A. Ribeiro and G.B. Giannakis, "Bandwidthconstrained distributed estimation for wireless sensor networks-part I: Gaussian case," IEEE Transactions on Signal Processing, vol. 54, no. 3, pp. 1131-1143, Mar. 2006.

[2] Z. Luo, "An isotropic universal decentralized estimation scheme for a bandwidth constrained ad hoc sensor network," IEEE Journal on Selected Areas in Communications, vol. 23, no. 4, pp. 735-744, Apr. 2005.

[3] H. C. Papadopoulos, G. W. Wornell, and A. V. Oppenheim, "Sequential signal encoding from noisy measurements using quantizers with dynamic bias control," IEEE Transactions on Information Theory, vol. 47, no. 3, pp. 978-1002, Mar. 2001.

[4] F. A. Shah, A. Ribeiro, and G. B. Giannakis, "Bandwidth-constrained MAP estimation for wireless sensor networks," in Proc. of 39th Asilomar Conference on Signals, Systems and Computers, Pacific Grove, CA, Oct. 28-Nov. 1, 2005, pp. 215-219.

[5] W. Lam and A. R. Reibman, "Design of quantizers for decentralized estimation systems," IEEE Transactions on Communications, vol. 41, no. 11, pp. 1602-1605, Nov. 1993.

[6] J. A. Gubner, "Distributed estimation and quantization," IEEE Transactions on Information Theory, vol. 39, no. 4, pp. 1456-1459, Jul. 1993.

[7] A. Ribeiro, G. B. Giannakis, and S. I. Roumeliotis, "SOI-KF: Distributed kalman filtering with low-cost communications using the sign of innovations," IEEE Transactions on Signal Processing, vol. 54, no. 12, pp. 4782-4795, Dec. 2006.

[8] E. J. Msechu, S. I. Roumeliotis, A. Ribeiro, and G. B. Giannakis, "Decentralized quantized kalman filtering with scalable communication cost," IEEE Transactions on Signal Processing, vol. 56, no. 8, pp. 3727-3741, Aug. 2008.

[9] N. Trawny, Cooperative localization: On motioninduced initialization and joint state estimation under communication constraints, Ph.D. thesis, University of Minnesota, Minneapolis, MN, Aug. 2010.

[10] N. Trawny and S. I. Roumeliotis, "Cooperative localization under communication constraints," Tech. Rep., Dept. of Comp. Sci. \& Eng., University of Minnesota, 2008, [Online] Available: http: //www-users.cs.umn.edu/ trawny/ Publications/Trawny_TR_Quant.pdf.
[11] N. Trawny, S. I. Roumeliotis, and G. B. Giannakis, "Cooperative multi-robot localization under communication constraints," in Proc. of the IEEE International Conference on Robotics and Automation, Kobe, Japan, May 12-17, 2009, pp. 4394-4400.

[12] E. D. Nerurkar, K. X. Zhou, and S. I. Roumeliotis, "A hybrid estimation framework for cooperative localization under communication constraints," in Proc. of the IEEE/RSJ International Conference on Intelligent Robots and Systems, San Francisco, CA, Sep. 25-30, 2011, pp. 502-509.

[13] E. D. Nerurkar and S. I. Roumeliotis, "Resourceaware hybrid esimation framework for multirobot cooperative localization," Tech. Rep., Dept. of Comp. Sci. \& Eng., University of Minnesota, 2012, [Online] Available: http: //www-users.cs.umn.edu/ nerurkar/ Nerurkar_multibithybridCL.pdf.

[14] P. S. Maybeck, Stochastic Models, Estimation, and Control, vol. 1, Academic Press, New York, 1979.

[15] G. H. Golub and C. F. Van Loan, Matrix Computations, Johns Hopkins University Press, 1983.

[16] S. Lloyd, "Least squares quantization in pcm," IEEE Transactions on Information Theory, vol. 28, no. 2, pp. 129-137, Mar. 1982.

[17] J. Max, "Quantizing for minimum distortion," IRE Transactions on Information Theory, vol. 6, no. 1, pp. 7-12, Mar. 1960.

[18] A. Prekopa, "Logarithmic concave measures and related topics," in Stochastic Programming. 1980, pp. 63-82, Academic Press.

[19] S. Boyd and L. Vandenberghe, Convex Optimization, Cambridge University Press, New York, NY, 2004.

[20] Y. Bar-Shalom and X. Li, Estimation and Tracking: Principles, techniques and software, Norwood, MA: Artech House, Inc., 1993. 